\title{
Resident Skills Assessment in Corneal Suturing: A Comprehensive Review of Currently Proposed Educational Programs and Evaluation Tools
}

\author{
Lea Dormegny, $\mathrm{MD}^{1,2}$ Nicole Neumann, $\mathrm{PhD}^{2}$ Anne Lejay, MD, PhD ${ }^{2,3}$ Arnaud Sauer, MD, $\mathrm{PhD}^{1,2}$ \\ David Gaucher, MD, PhD ${ }^{1,2}$ Nabil Chakfe, MD, PhD ${ }^{2,3}$ Tristan Bourcier, MD, PhD ${ }^{1,2}$
}

1 Department of Ophthalmology, New Civil Hospital, Strasbourg
University Hospital, Strasbourg, France
${ }^{2}$ GEPROVAS (Groupe Européen de Recherche sur les Prothèses
Appliquées à la Chirurgie Vasculaire), Department of
Anesthesiology, Civil Hospital, Strasbourg, France
${ }^{3}$ Department of Vascular Surgery and Kidney Transplantation,
Strasbourg University Hospital, France

J Acad Ophthalmol 2021;13:e304-e310.

\begin{abstract}
Address for correspondence Lea Dormegny, MD, Centre Hospitalier Universitaire de Strasbourg, 1 place de l'Hôpital, 67000 Strasbourg, France (e-mail: leadormegny@hotmail.fr).
\end{abstract}

\begin{abstract}
Purpose This study aimed to perform a comprehensive review of publications proposing educational programs for resident skills assessment in corneal suturing. Methods An extensive online article search in PubMed following the Preferred Reporting Items for Systematic Reviews and Meta-Analyses (PISMA) reporting guidelines was performed to identify prospective comparative studies or prospective before/after studies published up to March 2021 and reporting the assessment of ophthalmology residents' skills in corneal suturing during dedicated training sessions. Results Three studies were identified for review. The first reported the efficiency of an electromagnetic tracking system placed on the surgeon's fingers coupled with a computer analysis of movements and time to identify surgeons with different backgrounds in corneal suturing. The second reported the efficiency of the reference system in assessing the improvement of corneal suturing conducted by residents after a training session, with video-based assessment for economy and confidence of movement, limiting tissue damage and precision of operative technique, reviewed by blind assessors. The third proposed an innovative remote corneal suturing training method using Zoom for direct feedback to the residents. The stitch quality was assessed for length, depth, radiality, and tension. The results were similar when compared with a group of residents without feedback.

\section{- corneal suture \\ - training \\ - education \\ - assessment \\ - residents}

Keywords

Conclusion This review underlines the rarity and disparity of available tools for corneal suturing assessment, justifying the need for more complete models to be designed. These should consider body ergonomics and stitch quality and time. Comparative studies involving novices and attendings may provide reliable evaluation of existing gaps and specific metrics to target, helping residents to approach their superiors' experience.
\end{abstract}

received

June 2, 2021

accepted after revision

August 19, 2021
DOI https://doi.org/ 10.1055/s-0041-1740065. ISSN 2475-4757.

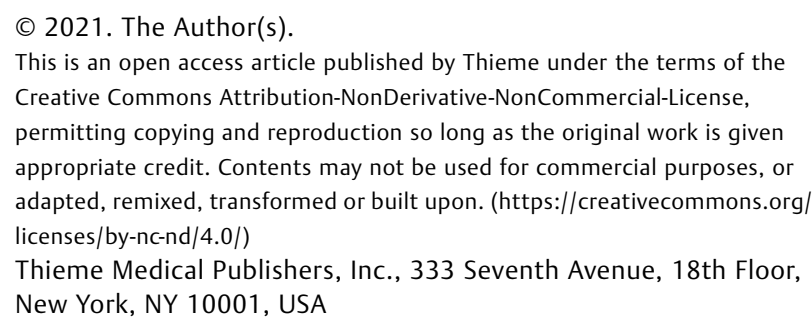

This is an open access article published by Thieme under the terms of the Creative Commons Attribution-NonDerivative-NonCommercial-License, permitting copying and reproduction so long as the original work is given appropriate credit. Contents may not be used for commercial purposes, or adapted, remixed, transformed or built upon. (https://creativecommons.org/ licenses/by-nc-nd/4.0/)

Thieme Medical Publishers, Inc., 333 Seventh Avenue, 18th Floor, New York, NY 10001, USA 
Corneal suturing is a fundamental step in ophthalmic surgery. While being part of regular elective surgeries (i.e., keratoplasties, amniotic membrane transplantations, and pterygium surgeries), it is also a key step in many urgent procedures, such as traumatic corneal wound closure. Since both of these situations might be encountered by novice surgeons, anticipatory training for corneal suturing during residency is mandatory to improve surgical outcomes and reduce the risk of complications in these procedures. ${ }^{1,2}$

The widespread use of surgical skills training on simulated tissue instead of actual patients has been thought to improve the learning curve of residents. Indeed, residents' evaluation during one specific procedure in the operating room $(\mathrm{OR})$ might vary according to the intrinsic difficulty of the procedure and the degree of stress affecting either the resident or the supervisor hampering a smooth procedure. Thus, reproducibility of the assessments in these conditions might be difficult., ${ }^{3,4}$ Many studies have reported the outcomes of penetrating keratoplasty procedures performed by residents during elective surgery in the $O \mathrm{R}^{5-11}$ However, a large diversity of assessment parameters has been proposed (i.e., details on surgery times, graft clarity, postoperative astigmatism, best corrected visual acuity, and graft survival), reflecting the difficulty of finding widely accepted and reproducible assessments for the quality of a given surgical procedure. Defining the optimal parameters for assessing good surgical procedures remains difficult, ${ }^{12}$ and the corresponding objective scores for their assessment in corneal suturing still need to be determined.

It is acknowledged that the repetition of one particular technique is key to acquiring and maintaining a high level of skill for surgeons. ${ }^{13}$ This evidence supports training programs. There is, however, a lack of data on how courses should be structured to promote the acquisition of skills. ${ }^{2}$ It remains difficult to design a dedicated training program that presents both relevant conditions and the right evaluation of specific surgical skills, ${ }^{14}$ such as corneal suturing. In addition, evaluations should indicate which specific task needs improvement, ${ }^{15,16}$ without indicating whether the given improvement will impact performance in the OR.

This study aims to conduct a comprehensive review of all publications proposing educational programs and assessment scores for ophthalmology residents dealing specifically with corneal suturing.

\section{Methods}

\section{Outcome}

The aim of this study was to review all available papers concerning the assessment of corneal suturing in residents during specific training sessions with the objective of improving their surgical skills. The surgical model, assessment tool, and training conditions proposed were defined.

\section{Search Strategy and Data Extraction}

A comprehensive search strategy was designed to retrieve all articles published up to March 2021 by combining (corneal suture OR corneal suturing OR corneal transplantation AND training OR residents OR novice surgeons OR assessment OR skill assessment OR quality assessment) in the electronic bibliographic database of PubMed and following the Preferred Reporting Items for Systematic Reviews and Meta-Analyses (PRISMA) reporting guidelines. All studies proposing an English abstract were reviewed. Inclusion criteria involved the study design (i.e., prospective comparative studies with two or more groups or prospective studies with before/after comparison [simple or crossover] in one single group), the type of population studied (i.e., ophthalmology residents or novice surgeons), and the study outcome (i.e., assessment of residents' surgical performance in corneal suturing during specific training sessions). All studies presenting with the following criteria were excluded from this review: inappropriate study type (i.e., retrospective studies, observational studies, clinical guidelines, consensus documents, reviews, systematic reviews, and conference proceedings), studies dealing with surgical procedures other than corneal suturing or with populations other than residents or novice surgeons or with another condition than training session (i.e., OR assessments) and, finally, studies reporting molecular aspects (i.e., artificial cornea design). The search results were compiled using EndNote X9.3.3 software. Two experienced reviewers screened the retrieved articles and extracted data from each eligible study using a standardized data extraction sheet following presence or absence of inclusion and exclusion criteria.

\section{Relevant Studies Analyses}

For each selected study, first author, publication date, study type, and country were retrieved. The following information on the methods was reported: defined groups, number of participants per group, eye support and type of sutures used, type of exercise assessed, assessment tool selected for the objective evaluation, surgical skills assessed, and training conditions. The outcomes for each study were also reported for further discussion.

\section{Results}

The database identified 175 articles. After removing 23 duplicates, 152 articles were reviewed based on the title and abstract. Of these, 148 were excluded based on study type (i.e., retrospective, observational, clinical guidelines, consensus documents, reviews, systematic reviews, and conference proceedings), surgical procedures other than corneal suturing, participants other than residents, surgical conditions other than training, and studies only reporting molecular aspects of corneal models. Four articles were assessed for the systematic review (-Fig. 1). One was excluded after a full-text review as it reported the same training session from the same team as another already included article, the difference being the results focused exclusively on ophthalmic surgeries other than corneal suturing. ${ }^{17}$ - Table 1 gives an overview of the methods and results of the three remaining studies. 


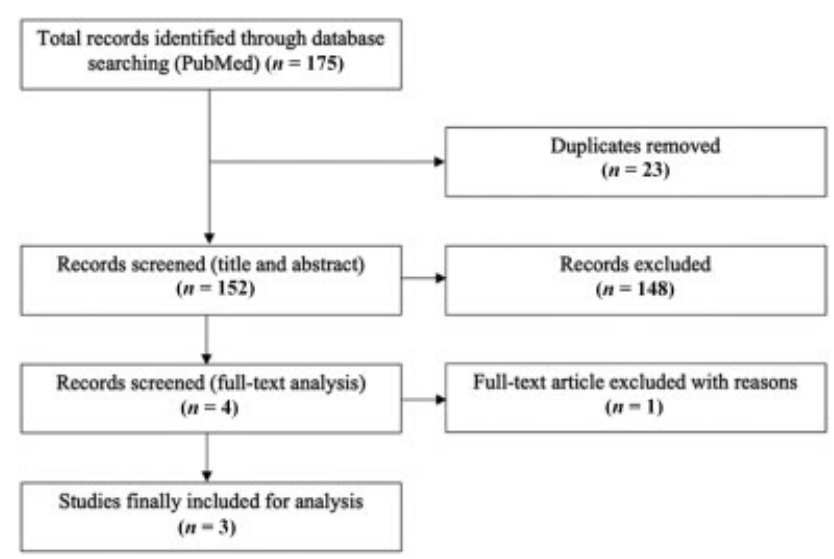

Fig. 1 Flow chart representing study selection.

\section{Study 1}

Study 1 was conducted in 2006 by Saleh et al. ${ }^{16}$ This prospective and comparative study included 30 surgeons assigned to three different groups according to their level of experience in corneal suturing (defined by the number of stitches previously performed). The surgeons were asked to perform a 10-0 nylon suture pass using a 3-1-1 pattern to close a wound on an artificial cornea under a microscope. Evaluation of the suture pass was performed using the Imperial College Surgical Assessment Device (ICSAD). Briefly, this method uses a 10-mm electromagnetic tracker attached to the surgeon's index finger of each hand from which threedimensional records are created using a magnetic field. The data were then analyzed using a custom-made computer software program which assessed the number of movements, the path length of these movements, and the time taken to complete the procedure.

The study showed that all three collected data points (i.e., number of finger movements, path length of these movements, and time taken to complete the task) significantly differed between groups. For each comparison, the more experienced group demonstrated fewer hand movements, shorter path length, and shorter time to perform the task, compared with the less experienced groups. Moreover, as experience increased during training, there was a reduction in the variability between the individuals within the cohort.

\section{Study 2}

Study 2 was conducted by Ezra et al in 2009. ${ }^{15}$ This prospective study included 20 residents assessed using ICSAD for one unique 10-0 nylon corneal stitch on a model eye with a microscope. All participants had to perform a stitch 1 week before and 1 week after a 1 -day surgical course, with the aim of measuring the impact of the course on corneal suturing. Also, a video-based system (i.e., the modified Objective Structured Assessment of Technical Skill [OSATS]) was evaluated and compared with ICSAD as a supplementary assessment tool. The OSATS videos were scored by two masked independent observers for economy of movement, confidence of movement, limiting tissue damage, and precision of operative technique on a 5-point Likert's scale. The 1-day course was precisely scheduled and included different teach- ing techniques, such as lectures for basic techniques on knot tying and instrument handling, along with practicing diverse surgical procedures including corneal suturing on porcine eyes.

This study showed that the three ICSAD parameters drastically improved after the 1-day course. When participants were divided into two experience-based groups, the improvement was more significant in the "senior group" than the "junior group." OSATS had good interrater reliability and was significantly better after training. There was significant correlation between two scores.

\section{Study 3}

Study 3 was conducted in 2020 by Pasricha et al. ${ }^{18}$ This prospective and comparative study included 10 residents randomly assigned to two groups and stratified by their postgraduate year. Participants were asked to perform four cardinal corneal stitches using 10-0 nylon on porcine eyes under a microscope just after a full-thickness trephination. All had a prewet laboratory virtual lecture reviewing proper corneal suturing technique. This exercise was then performed under two different conditions. During one remote training exercise, residents' hand and body positioning, as well as their microscopic view were both remotely transmitted to a virtual whiteboard conference with three attending surgeons using Zoom. The attendings could give direct oral and gestural feedback to the residents. One other training condition consisted of performing the same task without any feedback. The porcine eyes were scored at the end of the session by two masked graders using the modified rubric from the Mayo Clinic corneal trauma curriculum to provide a maximum score of 6 points per suture pass ( 24 points per trial). The six items evaluated were as follows: (1) stitch length in the graft, (2) stitch length in the host, (3) stitch depth in the graft, (4) stitch depth in the host, (5) stitch radiality, and (6) stitch tension. During the first evaluation, one group (remote $[+]$ ) used remote training while the other (remote $[-]$ ) used training without feedback. Both groups then performed one training exercise without feedback and, finally, the remote (-) group underwent remote training and the remote $(+)$ group the training exercise without feedback. Additionally, pre- and postwet laboratory surveys were completed by the residents to assess their confidence with corneal suturing and postwet laboratory surveys were completed by both the residents and attendings to assess the effectiveness of the remote wet laboratory.

Both groups had similar scores for corneal suturing during all evaluations. Only the depth of the stitch was better in the remote $(+)$ group after the first trial. First-year residents performed similarly to other residents. All residents' rates for comfort with corneal suturing increased significantly after the remote wet laboratory. The remote wet laboratory was considered highly effective and at least as effective as previous in-person wet laboratories by residents and attendings. Attendings rated the remote wet laboratory as highly effective in evaluating all six components, with suture depth being the lowest rated. Finally, the prewet laboratory virtual lecture was rated highly effective by all residents and the 


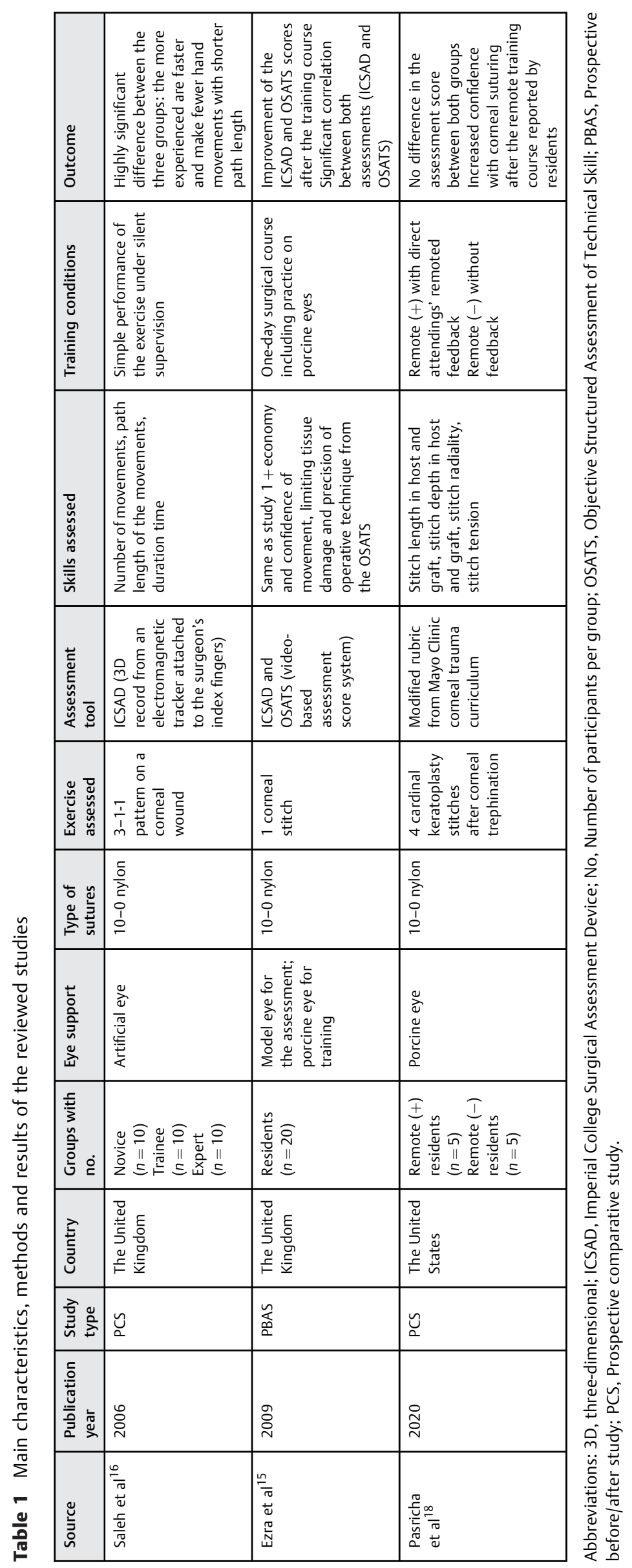


virtual whiteboard during the remote wet laboratory considered highly effective by the residents and attendings.

\section{Discussion}

\section{General Considerations}

This study aimed to review the parameters proposed for residents' assessment in corneal suturing during devoted training sessions. In this review, only three studies, from two countries, were relevant considering our inclusion criteria. In these studies, only three different assessment tools were reported. Even if our inclusion criteria might have been restrictive, we believe that a prospective comparative study (either with group comparison or before/after comparison) is a mandatory design for the evaluation of such assessment tools. This small number of studies is not in line with the substantial need for training in the field of ophthalmic surgery, as many ophthalmology residents still struggle with surgical procedures. ${ }^{19}$

\section{Video Assessment by Attendings}

In study $2,{ }^{15}$ the authors describe a video-recorded assessment, the OSATS, scored by two masked independent observers for economy of movement, confidence of movement, limiting tissue damage, and precision of operative technique on a 5-point Likert's scale. This tool was efficient in showing an improvement of these skills after a 1-day training course, using the performance of one corneal stitch as the assessment exercise. Video-recorded assessment affords the possibility of reviewing the video at the assessor's convenience and ensures masked evaluation. However, some aspects of the "live" surgical procedure might be missed, such as wholebody coordination (for instance, head movements out of the microscope to be combined with needle loss). Moreover, OSATS focuses on the ergonomic performance of the surgeon, which does play a great part in the quality of the procedure, ${ }^{20}$ yet limited focus is thus paid to the quality of the stitch (depth and tension of the stitch, for instance, are not assessed). Also, one could wonder if one corneal stitch is enough to assess surgical skill improvement after training. Indeed, more elaborate tasks might be needed for this assessment. Interestingly, the improvement_of corneal suturing was more significant in the "senior group" than in the "junior group" which could suggest either that basic experience in surgery gives more possibilities for improvement, or that the assessment tool was not realistic enough to differentiate novices' improvement from that of more experienced trainees. ${ }^{15}$

Remote assessment is possible using video-recorded evaluations. In study 3 , for example, real-time remote assessment and teaching was performed by attendings while visualizing residents' performance on four cardinal corneal sutures in porcine eyes. ${ }^{18}$ Such training was conducted using everyday equipment (i.e., laptop and smartphone) and the Zoom program which makes it easy to reproduce. Moreover, the residents and attendings rated the remote training program as highly effective for real-time teaching. The use of a virtual white board might have actually provided ergo- nomic and comprehensive viewing of the exercise. However, objective assessments of stitch quality (i.e., by depth, length, tension, and radiality) were similar between the residents with remote feedback and those without. Also, when comparing residents according to their postgraduate years, stitch quality remained similar. While proposing a longer exercise for suturing than study 2 , this study nevertheless failed to demonstrate the effectiveness of remote training in skill improvement. Of note, no evaluation of residents' ergonomic and posture was proposed which might have been helpful either for a before/after comparison or for a comparison according to the level of experience. An evaluation via Zoom may also be limited as transmission of the microscope autofocus was not possible, thus constraining the remote evaluation. ${ }^{18}$ For both study 2 and study 3 , time to perform suture pass and additional orientations (i.e., within 1 clock hour) may have added value to the assessment as these are important in clinical practice. ${ }^{15,18}$

\section{Computerized Assessment with Automated Feedback} Another type of assessment tool was described in study $1 .{ }^{16}$ The ICSAD motion analysis system was used to evaluate participants practicing a 3-1-1 pattern corneal suture. This tool was able to differentiate between participants according to their level of experience by automatically measuring their hand movements, the path length of these movements, and time to perform the task. Moreover, as experience increased over the course of training, the value variability between individuals within the cohort decreased. In fact, one advantage of motion analysis is to provide direct feedback on movement efficiency which helps trainees move toward the values obtained by more experienced surgeons. Such autoevaluation does not require the intervention of an attending and might motivate trainees to improve their scores on their own. However, to be installed, the ICSAD system needs to be manipulated which increases the risk of incorrect setup and, subsequently, distorted results, especially in the absence of a supervisor. Its design might also impede a surgeon's movement during microsurgical procedures as it is cumbersome. ${ }^{15}$ Finally, the equipment needed is expensive. ${ }^{15}$ Unfortunately, this study did not assess stitch quality and the 3-1-1 pattern proposed is not universally used for corneal wound closure which might hamper its reproducibility. Nevertheless, this is the only study proposing a comparison between surgeons with different levels of experience in corneal suturing, and it defines some parameters derived from movement efficiency that might differentiate between attendings' and novices' performances. This creates an opening for future research to enlarge the field of parameters for such differentiation and determine specific activities to narrow the gap between novices and attendings.

Other automated feedback tools have been described in ophthalmic surgery, are not yet validated: optic fiber on a patch to be pressed and pierced by wound stitches for strain measurement and anterior segment optical coherence tomography real-time feedback during procedures involving the anterior segment of the eye. ${ }^{21,22}$ 


\section{Innovative Training Modalities}

The importance of verbal communication during teaching has been highlighted in studies including attending supervision. In study 2, a 1-day course with very precise schedule was used, alternating lectures with practical sessions and feedback sessions. The authors described a behavioral and cognitive learning approach in which the teachers organize their teaching to mirror the internal learning process of their students. ${ }^{15}$ This type of training was designed according to Gagné's instructional model and aims to end passive learning sessions which should become more interactive and "linked to external benchmarks" with a more efficient and costeffective educational effort. ${ }^{17,23}$ Also, in study $3,^{18}$ verbal explanations were proposed to all residents to remind them of the process of corneal suturing which the residents rated as very helpful.

\section{Future Assessment Tools}

Through the review of these three studies, we pointed out the rarity of available tools for corneal suture assessment in residents. Moreover, none of the assessments assessed here reflected both stitch quality and the overall ergonomics of the procedure, while all were used for the evaluation of relatively short exercises. There is evidence that more complete tools are required for such assessment. We believe that autofeedback devices have a great potential for objective and reproducible assessments of residents' skills while being a source of motivation and self-education for the residents. However, it is mandatory to find a cost-effective and easy-tomanipulate device which should be able to assess diverse metrics like body and head position, hand movements, and stitch quality. Finally, surgical procedure time also defines its quality and should be measured as well. ${ }^{20}$ Although such a complex device does not exist yet, quite comprehensive corneal suturing assessment scales already exist and might be considered for the design of a more complete assessment method. ${ }^{24,25}$

While diverse cornea models were used in this review, there have been several other models tested for training in corneal suturing. ${ }^{26,27}$ Having one universally defined model might be of great help to ensure comparability between published results and should be designed as a close-toreality corneal model.

\section{Conclusion}

Finally, this review underlines the great need for new metrics to be defined for the assessment of corneal suturing in ophthalmology. One reproducible tool might be tricky to elaborate without knowing which parameters differentiate novice from attending surgeons' performance. To define such metrics, prospective and comparative studies between novices and attendings should be the chosen design for reliable evaluation of those existing gaps. Specific points to work on, helping residents to approach their superiors' experience, should consider timing, stitch quality, and ergonomics for assessment parameters.
Funding

No funding source was used for this study.

Conflict of Interest

None declared.

\section{References}

1 Le K, Bursztyn L, Rootman D, Harissi-Dagher M. National survey of Canadian ophthalmology residency education. Can J Ophthalmol 2016;51(03):219-225

2 ACGME. Accreditation Council for Graduate Medical Education. Accreditation Council for Graduate Medical Education Web site. Accessed November 2020 at: http://www.acgme.org

3 Barnes RW. Surgical handicraft: teaching and learning surgical skills. Am J Surg 1987;153(05):422-427

4 Aggarwal R, Boza C, Hance J, Leong J, Lacy A, Darzi A. Skills acquisition for laparoscopic gastric bypass in the training laboratory: an innovative approach. Obes Surg 2007;17(01):19-27

5 Randleman JB, Song CD, Palay DA. Indications for and outcomes of penetrating keratoplasty performed by resident surgeons. Am J Ophthalmol 2003;136(01):68-75

6 Wiggins RE, Cobo M, Foulks GN. Results of penetrating keratoplasty by residents. Arch Ophthalmol 1990;108(06):851-853

7 Kutzscher EM, Sorenson AL, Goodman DF. Penetrating keratoplasty performed by residents. Arch Ophthalmol 2004;122(09): 1333-1336

8 Shimmura-Tomita M, Shimmura S, Tsubota K, Shimazaki J. Penetrating keratoplasty performed by residents compared with an experienced cornea transplant surgeon. J Surg Educ 2017;74(02): 258-263

9 Mascaro VL, Scarpi MJ, Hofling-Lima AL, de Sousa LB. Corneal transplantation in keratoconus: evaluation of results and complications obtained by skillful and surgeons in training [in Portuguese]. Arq Bras Oftalmol 2007;70(03):395-405

10 Gross RH, Poulsen EJ, Davitt S, Schwab IR, Mannis MJ. Comparison of astigmatism after penetrating keratoplasty by experienced cornea surgeons and cornea fellows. Am J Ophthalmol 1997; 123(05):636-643

11 Hammoudi DS, Segev F, Abdolell M, Rootman DS. Outcome of penetrating keratoplasty performed by cornea fellows compared with that of an experienced staff surgeon. Cornea 2005;24(04): 410-416

12 Baldwin PJ, Paisley AM, Brown SP. Consultant surgeons' opinion of the skills required of basic surgical trainees. Br J Surg 1999;86 (08):1078-1082

13 Hamstra SJ, Dubrowski A, Backstein D. Teaching technical skills to surgical residents: a survey of empirical research. Clin Orthop Relat Res 2006;449(449):108-115

14 Thomsen AS, Subhi Y, Kiilgaard JF, la Cour M, Konge L. Update on simulation-based surgical training and assessment in ophthalmology: a systematic review. Ophthalmology 2015;122(06): 1111-1130.e1

15 Ezra DG, Aggarwal R, Michaelides M, et al. Skills acquisition and assessment after a microsurgical skills course for ophthalmology residents. Ophthalmology 2009;116(02):257-262

16 Saleh GM, Voyatzis G, Hance J, Ratnasothy J, Darzi A. Evaluating surgical dexterity during corneal suturing. Arch Ophthalmol 2006;124(09):1263-1266

17 Koukkoulli A, Chandra A, Sheth H, et al. Bridging the gap: theorybased design of a microsurgical skills course for ophthalmology residents. J Surg Educ 2015;72(04):585-591

18 Pasricha ND, Haq Z, Ahmad TR, et al. Remote corneal suturing wet lab: microsurgical education during the COVID-19 pandemic. J Cataract Refract Surg 2020;46(12):1667-1673 
19 Binenbaum G, Volpe NJ. Ophthalmology resident surgical competency: a national survey. Ophthalmology 2006;113(07): 1237-1244

20 Ellis H. Famous Operations. New York, NY: Media, Pa: Harwal Pub. Co.; 1984

21 Handelman A, Keshet Y, Livny E, Barkan R, Nahum Y, Tepper R. Evaluation of suturing performance in general surgery and ocular microsurgery by combining computer vision-based software and distributed fiber optic strain sensors: a proof-of-concept. Int J CARS 2020;15(08):1359-1367

22 Todorich B, Shieh C, DeSouza PJ, et al. Impact of microscopeintegrated OCT on ophthalmology resident performance of anterior segment surgical maneuvers in model eyes. Invest Ophthalmol Vis Sci 2016;57(09):OCT146-OCT153

23 Lee AG, Oetting T, Beaver HA, Carter KTask Force on the ACGME Competencies at the University of Iowa Department of Ophthal- mology. The ACGME Outcome Project in ophthalmology: practical recommendations for overcoming the barriers to local implementation of the national mandate. Surv Ophthalmol 2009;54(04):507-517

24 Zhang Z, Zhou M, Liu K, et al. Development of a new valid and reliable microsurgical skill assessment scale for ophthalmology residents. BMC Ophthalmol 2018;18(01):68

25 Golnik KC, Motley WW, Atilla H, et al. The ophthalmology surgical competency assessment rubric for strabismus surgery. J AAPOS 2012;16(04):318-321

26 Pujari A, Sharma N, Chaniyara $\mathrm{MH}$, et al. Optimal refinement of residents' surgical skills by training on induced goat's eye corneoscleral perforation. Indian J Ophthalmol 2019;67(04): 547-548

27 Gurbaxani A, DeCock R. Anyone for leftovers? A simple method for teaching corneal graft suturing. Cornea 2010;29(09):1072 